\title{
Checklist of the Holothuroidea (Echinodermata) from the State of Paraíba, Brazil
}

\author{
Jéssica Prata ${ }^{1} \&$ Martin Lindsey Christoffersen ${ }^{1,2}$
}

(1) Universidade Federal da Paraíba, Departamento de Sistemática e Ecologia, Programa de Pós-Graduação em Ciências Biológicas, Cidade Universitária, João Pessoa 58051-900, Paraíba, Brazil. E-mail: jessie.prata@gmail.com

(2) Universidade Federal da Paraíba, Departamento de Sistemática e Ecologia, Laboratório de Biodiversidade de Invertebrados não-Insetos, Cidade Universitária, João Pessoa 58051-900, Paraíba, Brazil. E-mail: mlchrist@dse.ufpb.br

Prata J. \& Christoffersen M.L. (2017) Checklist of the Holothuroidea (Echinodermata) from the State of Paraíba, Brazil. Pesquisa e Ensino em Ciências Exatas e da Natureza, 1(1): 45-59.

\section{Checklist de Holothuroidea (Echinodermata) do Estado da Paraíba, Brasil}

Resumo: O estudo apresenta a primeira lista de espécies de holotúrias para a costa do Estado da Paraíba, região nordeste do Brasil. A lista de espécies foi elaborada com base no estudo de espécimes depositados na coleção de Invertebrados Paulo Young, Universidade Federal da Paraíba. Um total de 16 espécies pertencentes a sete famílias foram registradas. Dessa forma, contribuímos para os estudos de biodiversidade da costa nordeste do Brasil.

Palavras chave: Pepinos-do-mar, Taxonomia, nordeste do Brasil, Oceano Atlântico.

Abstract: The study presents the first species list for holothurians from the coast of the State of Paraíba, northeastern Brazil. The list was made based on the study of specimens deposited in the Invertebrate Collection Paulo Young, Federal University of Paraíba. A total of 16 species from seven families of Holothuroidea were recorded. This paper contributes to the biodiversity studies from the northeast coast of Brazil.

Key words: Sea cucumbers, Taxonomy, northeastern Brazil, Atlantic Ocean.

\section{Introduction}

Holothurians constitute one of the most abundant animal groups in sea bottoms, where they may represent 95\% of the total biomass in deep regions (Heezen \& Hollister 1971). In Brazil, about 50 species of Holothuroidea have been recorded (Ventura et al. 2012).

Studies dealing mainly with members of the class Holothuroidea are relatively scarce in Brazil. Ludwig (1881) cites species from northeastern Brazil in his list of Brazilian coastal holothurians. Sluiter (1910) published a checklist of sea cucumbers from the West Indies, including some species recorded from northeastern Brazil. Ancona Lopez \& Sawaya (1955) recorded holothurian species from Recife (State of Pernambuco). Deichmann (1963) studied holothurians from Caribbean waters and also recognized some species reported from northeastern Brazil. Tommasi (1969) lists the Brazilian species of Holothuroidea, including material from the northeastern coastline. Recent papers dedicated to the knowledge of the Brazilian Holothuroidea, such as Martins et al. (2012a, 2012b, 2017), Prata \& Christoffersen (2012, 2016), Prata et al. (2014a, 2014b, Martins \& Souto (2015) and Moura et al. (2015) added a significant number of species, including new records and new species. 
According to Lessius (2005), faunistic inventories containing new species identifications are indispensable, because they subsidize the comprehension of structure, dynamics and diversity of local communities. Inventories represent the basis for biodiversity knowledge. They present data on species identification, distribution, and abundance, providing elements in support of conservation measures and for the sustainable use of the natural environment (Amaral \& Jablonski 2005).

The study aimed to present the first list of species of Holothuroidea for the coast of the State of Paraíba.

\section{Material and Methods}

\section{Study site}

The State of Paraíba has approximately $138 \mathrm{~km}$ of coastline which corresponds to about $4.6 \%$ of the northeast coast (De Assis et al. 2012) and considerable diversity of coastal ecosystems (Araújo et al. 2008; De Assis et al. 2012; Lima et al. 2014, 2017). The reef structures in coast of the State of Paraíba occur in the north from the littoral near the mouth of the Mamanguape River to Baía da Traição, until the south near the estuary of the Paraíba River up to the limits with State of Pernambuco (Costa et al. 2007). Along its length there are environments ranging from reefs to estuaries, with many types of beaches, but predominating those with arenitic formations (Paraiba 2000).

\section{Data collection and analysis}

The present checklist was based on species of Holothuroidea deposited in the Invertebrate Collection Paulo Young, Federal University of Paraíba. The specimens were collected along the coast of Paraíba.

Taxonomic list was organized systematically according Appeltans et al. (2012). The methods used for identification followed those of Rowe \& Doty (1977) and Pawson et al. (2010). Tissue fragments from tentacles, body wall, papillae and tube feet were immersed in a $3 \%$ solution of sodium hypochlorite and washed in five changes of distilled water and after in five changes of absolute ethanol for the extraction of ossicles. Slides were prepared and observed under a light microscope. Photos of specimens were taken using a Canon Powershot A2000IS digital camera and a Leica MZ12.5 stereomicroscope.

Abbreviation used: UFPB/ECH, Echinodermata from Invertebrate Collection Paulo Young, Federal University of Paraíba; PB, Paraíba; spec., specimen(s).

\section{Results}

We analyzed 3.271 specimens and recorded 16 species, 13 genera, belonging to seven families and classified in three orders (Dendrochirotida, Aspidochirotida and Apodida) of holothurians for the coast of the State of Paraíba.

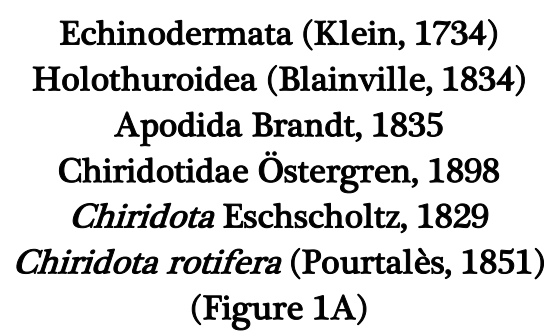

Material examined: UFPB/ECH-2127, 14 spec., Barra de Camaratuba, PB; UFPB/ECH-185, 3 spec., Praia do Cabo Branco, João Pessoa, PB; UFPB/ECH-186, 3 spec., Praia do Cabo Branco, João Pessoa, PB; UFPB/ECH-187, 3 spec., Praia do Cabo Branco, João Pessoa, PB; UFPB/ECH-188, 1 spec., Praia do Cabo Branco, João Pessoa, PB; UFPB/ECH-189, 2 spec., Praia do Cabo Branco, João 
Pessoa, PB; UFPB/ECH-190, 3 spec., Praia do Cabo Branco, João Pessoa, PB; UFPB/ECH-191, 9 spec., Praia do Cabo Branco, João Pessoa, PB; UFPB/ECH-192, 4 spec., Praia do Cabo Branco, João Pessoa, PB; UFPB/ECH-193, 15 spec., Praia do Cabo Branco, João Pessoa, PB; UFPB/ECH-194, 2 spec., Praia do Cabo Branco, João Pessoa, PB; UFPB/ECH-195, 2 spec., Praia do Cabo Branco, João Pessoa, PB; UFPB/ECH-196, 3 spec., Praia do Cabo Branco, João Pessoa, PB; UFPB/ECH-198, 3 spec., Praia do Cabo Branco, João Pessoa, PB; UFPB/ECH-199, 2 spec., Praia do Cabo Branco, João Pessoa, PB; UFPB/ECH-200, 1 spec., Praia do Cabo Branco, João Pessoa, PB; UFPB/ECH-853, 1 spec., Praia do Cabo Branco, João Pessoa, PB; UFPB/ECH-1068, 1 spec., Recifes do Seixas, João Pessoa, PB; UFPB/ECH-1220, 8 spec., Barra de Camaratuba, Mataraca, PB; UFPB/ECH-1468, 9 spec., Praia do Cabo Branco, João Pessoa, PB; UFPB/ECH-2123, 1 spec., Praia do Cabo Branco, João Pessoa, PB; UFPB/ECH-2126, 1 spec., Praia do Cabo Branco, João Pessoa, PB; UFPB/ECH2128, 6 spec., Ponta do Cabo Branco, João Pessoa, PB; UFPB/ECH-2129, 1 spec., Barra de Camaratuba, PB; UFPB/ECH-176, 1 spec., Praia do Cabo Branco, João Pessoa, PB; UFPB/ECH-177, 8 spec., Praia do Cabo Branco, João Pessoa, PB; UFPB/ECH-178, 3 spec., Ponta do Seixas, Cabo Branco, João Pessoa, PB; UFPB/ECH-179, 3 spec., 06 56' $847^{\prime \prime}$ S, 034'50'010" W, Porto de Cabedelo; UFPB/ECH-181, 2 spec., Arrecifes da Ponta do Seixas, João Pessoa, PB; UFPB/ECH-182, 1 spec., Ponta do Seixas, Cabo Branco, João Pessoa, PB; UFPB/ECH-1497, 5 spec., Praia do Cabo Branco, João Pessoa, PB; UFPB/ECH-1498, 1 spec., Recifes do Seixas, João Pessoa, PB; UFPB/ECH-1500, 1 spec., Pomar das Esponjas, João Pessoa, PB; UFPB/ECH-1953, 1 spec., Ponta do Cabo Branco, João Pessoa, PB; UFPB/ECH-1481, 6 spec., Praia do Maceiozinho, Jacumã, Conde, PB; UFPB/ECH-1950, 1 spec., Praia de Tambaba, Município Conde, PB; UFPB/ECH-1952, 1 spec., Recifes da Praia de Carapibus, Município Conde, PB; UFPB/ECH-1955, 2 spec., Praia de Pitimbú, Município Pitimbú, PB; UFPB/ECH-2039, 6 spec., Praia de Pitimbú, Município Pitimbú, PB; UFPB/ECH-2045, 1 spec., $07^{\circ} 03^{\prime} 48^{\prime S}$, 344ㄴ'00"W, PB; UFPB/ECH-2054, 6 spec., Praia de Pitimbú, Município Pitimbú, PB; UFPB/ECH-330, 3 spec., 07²28'01"S, 34048'47.35"W, Praia de Pitimbú, PB.

Type locality: Biscayne Bay, Florida (Deichmann 1930).

Diagnosis: Body wall thin, with numerous dispersed ossicles of the curved stem type. Whitish papillae are abundant on the body surface (Deichmann 1930). The species also has ossicles of the wheel type, with six rays (Miller \& Pawson 1984).

Geographic distribution: Florida, Mexico, Belize, Antilles, Puerto Rico, Bonaire and Brazil (Hendler et al. 1995).

\author{
Synaptidae Burmeister, 1837 \\ Synaptula Oersted, 1849 \\ Synaptula hydriformis (Lesueur, 1824)
}

(Figure 1B)

Material examined: UFPB/ECH-156, 1 spec., Picãozinho, João Pessoa, PB; UFPB/ECH-157, 1 spec., Picãozinho, João Pessoa, PB; UFPB/ECH-158, 1 spec., Picãozinho, João Pessoa, PB; UFPB/ECH-159, 2 spec., Picãozinho, João Pessoa, PB; UFPB/ECH-160, 1 spec., Recife de São Gonçalo, João Pessoa, PB; UFPB/ECH-161, 2 spec., Picãozinho, João Pessoa, PB; UFPB/ECH-162, 1 spec., Recife de Picãozinho, João Pessoa, PB; UFPB/ECH-163, 1 spec., Picãozinho, João Pessoa, PB; UFPB/ECH-164, 2 spec., Picãozinho, João Pessoa, PB; UFPB/ECH-165, 1 spec., Praia do Cabo Branco, João Pessoa, PB; UFPB/ECH-166, 2 spec., Picãozinho, João Pessoa, PB; UFPB/ECH-167, 2 spec., Picãozinho, João Pessoa, PB; UFPB/ECH-168, 4 spec., Picãozinho, João Pessoa, PB; UFPB/ECH-169, 2 spec., Picãozinho, João Pessoa, PB; UFPB/ECH-1501, 2 spec., Recifes do Seixas, João Pessoa, PB; UFPB/ECH-1554, 10 spec., Recifes do Cabo Branco, João Pessoa, PB; UFPB/ECH-1956, 1 spec., Recife do Seixas, João Pessoa, PB; UFPB/ECH-1958, 1 spec., Recife de São Gonçalo, João Pessoa, PB. 
Type locality: Guadeloupe (Lesueur 1824).

Diagnosis: Small, cylindrical form. Mouth anterior. Anus posterior. Tegument thin, of variable color. With 12 tentacles. Calcareous ring well developed. Radial plate with anterior border undulated and with a short but broad posterior "V"-shaped projection. Inter-radial plates with a slight anterior depression and a short posterior projection. Tentacular ampullae large. Two stone canals present. Two madreporites. One Polian vesicle. First intestinal loop connected to a mesenterium on the left side of the body wall. Ossicles of body wall anchor-shaped and attachment plates, including miliary granules (Laguarda-Figueras et al. 2001).

Geographic distribution: Florida, Antilles, Belize and Brazil (Hendler et al. 1995).

\author{
Aspidochirotida Grube, 1840 \\ Holothuriidae Ludwig, 1894 \\ Holothuria Linnaeus, 1767 \\ Holothuria (Thymiosycia) Pearson, 1914 \\ Holothuria (Thymiosycia) arenicola Semper, 1868 \\ (Figure 1C)
}

Material examined: UFPB/ECH-1480, 1 spec., Praia do Cabo Branco, João Pessoa, PB; UFPB/ECH697, 1 spec., Praia do Cabo Branco, João Pessoa, PB; UFPB/ECH-1062, 1 spec., Praia do Cabo Branco, João Pessoa, PB.

Type locality: Lighthouse Reef, Puerto Rico (Laguarda-Figueras et al. 2001).

Diagnosis: Body narrow, cylindrical, may attain about $20 \mathrm{~cm}$ in length. Tube feet distributed into indistinct bands, ventrally cylindrical, dorsally partially papilliform. Cuvier organs present. Most superficial body wall spicules are tables with four to eight marginal holes and short spire, often with slightly convergent pillars and few spines on top. Small, smooth buttons present, with about six perforations. Ventral podia with perforated tips. Dorsal podia with terminal plate smaller or entirely absent, and short bars, frequently curved. Color varies from grey to ferruginous red, with dark spots (Deichmann 1958).

Geographic distribution: Florida to Brazil (Hendler et al. 1995).

\author{
Holothuria (Halodeima) Pearson, 1914 \\ Holothuria (Halodeima) grisea Selenka, 1867 \\ (Figure 1D)
}

Material examined: UFPB/ECH-1962, 1 spec., Recife de São Gonçalo, João Pessoa, PB; UFPB/ECH-1072, 2 spec., Barra de Camaratuba-Mataraca, PB; UFPB/ECH-2141, 4 spec., Barra de Camaratuba, PB; UFPB/ECH-1793, 1 spec., Praia de Baía da Traição, Baía da Traição, PB; UFPB/ECH-1459, 1 spec., Recifes de Barra de Mamanguape, Rio Tinto, PB; UFPB/ECH-1516, 1 spec., Praia de Carapibus, Conde, PB; UFPB/ECH-1517, 1 spec., Praia de Coqueirinho, Conde, PB; UFPB/ECH-1222, 1 spec., Pier de Cabedelo, Cabedelo, PB; UFPB/ECH-1223, 1 spec., Barra de Camaratuba, Mataraca, PB; UFPB/ECH-439, 1 spec., Praia De Santa Catarina, Cabedelo, PB; UFPB/ECH-311, 1 spec., Praia do Cabo Branco, João Pessoa, PB; UFPB/ECH-316, 2 spec., Praia do Cabo Branco, João Pessoa, PB; UFPB/ECH-319, 2 spec., Praia do Seixas, João Pessoa, PB; UFPB/ECH-272, 2 spec., Praia do Cabo Branco, PB; UFPB/ECH-276, 1 spec., Ponta do Seixas, João Pessoa, PB; UFPB/ECH-277, 2 spec., Praia do Cabo Branco, PB; UFPB/ECH-278, 1 spec., Praia do Cabo Branco, PB; UFPB/ECH-207, 2 spec., Praia do Cabo Branco, João Pessoa, PB; UFPB/ECH-213, 1 spec., Ponta do Seixas, Cabo Branco, João Pessoa, PB; UFPB/ECH-313, 1 spec., Praia do Cabo Branco, João Pessoa, PB; UFPB/ECH-1141, 1 spec., Praia de Carapibus, Município do Conde, PB; 
UFPB/ECH-776, 1 spec., Praia de Coqueirinho, Conde, PB; UFPB/ECH-2145, 1 spec., Tabatinga, $\mathrm{PB}$.

Type locality: Haiti (Deichmann 1930).

Diagnosis: Length up to $25 \mathrm{~cm}$. Color distinct, with variable hues from bright red to yellow. Mouth positioned slightly ventral, with 20-25 peltate and branched tentacles. Ossicles of body wall are tables with approximately 12 marginal spines on disc. There is also an inner layer with plates with two or four central perforations, and the margins of the plates are equipped with robust spines (Pawson et al. 2010).

Geographic distribution: Gulf of Mexico, Antilles, Colombia, Puerto Rico, Venezuela, Brazil and West Africa (Pawson et al. 2010).

\title{
Holothuria (Cistypus) Haacke, 1880 \\ Holothuria (Cystipus) pseudofossor Deichmann, 1930
}

(Figure 1E)

Material examined: UFPB/ECH-2070, 1 spec., 06 57'S, 34³8'W, PB.

Type locality: Montego Bay, Jamaica (Deichmann 1930).

Diagnosis: Body wall not very thick, with small papillae on dorsal region, Tube feet not very numerous, cylindrical, distributed uniformly. Mouth ventral, surrounded by 20 tentacles. Anus terminal, without anal teeth. Spicules of body wall in the shape of tables with wavy margins, spires short, ending in a crown of small spines with a central perforation, surrounded by smaller perforations. Buttons with three or more pairs of perforations, with wavy margins. Tube feet with tables and plates. Dorsal papillae similar to those of body wall. Tentacles with spiny rods (based on description of Deichmann 1930).

Geographic distribution: Florida, Antilles, Puerto Rico, Curaçao, Venezuela and Brazil (Tommasi 1972; Hendler et al. 1995).

\author{
Dendrochirotida Grube, 1840 \\ Cucumariidae Ludwig, 1894 \\ Thyonidium Düben \& Koren, 1844 \\ Thyonidium seguroensis (Deichmann, 1930) \\ (Figure 1F)
}

Material examined: UFPB/ECH-2101, 5 spec., 07 03'48"S, 3445'10"W, PB; UFPB/ECH-2102, 1 spec., Barra de Camaratuba, Mataraca, PB; UFPB/ECH-2103, 2 spec., 0703'50"S, 3447'19"W, PB; UFPB/ECH-2104, 1 spec., 0704'24.4"S, 34²7'49"W, PB; UFPB/ECH-2065, 3 spec., 0704'51"S,

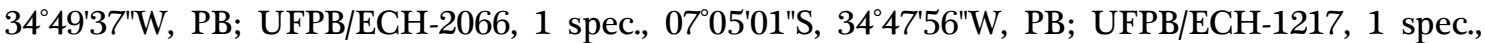
Recife de Areia Vermelha, Município de Cabedelo, PB; UFPB/ECH-2055, 2 spec., Recifes do Seixas, João Pessoa, PB; UFPB/ECH-2050, 2 spec., 0703'51"S, 3449'51"W, PB; UFPB/ECH-2151, 1 spec., Picãozinho, João Pessoa, PB; UFPB/ECH-2046, 1 spec., 0703'48"S, 34 $45^{\prime} \mathrm{W}$, PB; UFPB/ECH2047, 2 spec., 0705'05"S, 3444'21"W, PB; UFPB/ECH-2017, 1 spec., 0700 '26"S, 3449'25"W, Praia do Bessa, João Pessoa, PB; UFPB/ECH-1988, 1 spec., Praia de Tambaú, João Pessoa, PB; UFPB/ECH1997, 1 spec., Recife do Seixas, João Pessoa, PB; UFPB/ECH-1553, 1 spec., Recifes do Cabo Branco, João Pessoa, PB; UFPB/ECH-1551, 2 spec., Recifes do Cabo Branco, João Pessoa, PB; UFPB/ECH1680, 1 spec., Ponta do Cabo Branco, João Pessoa, PB; UFPB/ECH-874, 2 spec., Ponta do Seixas, João Pessoa, PB; UFPB/ECH-2040, 2 spec., Praia de Pitimbú, Município de Pitimbú, PB. 
Type locality: Porto Seguro, Bahia, Brazil (Deichmann 1930).

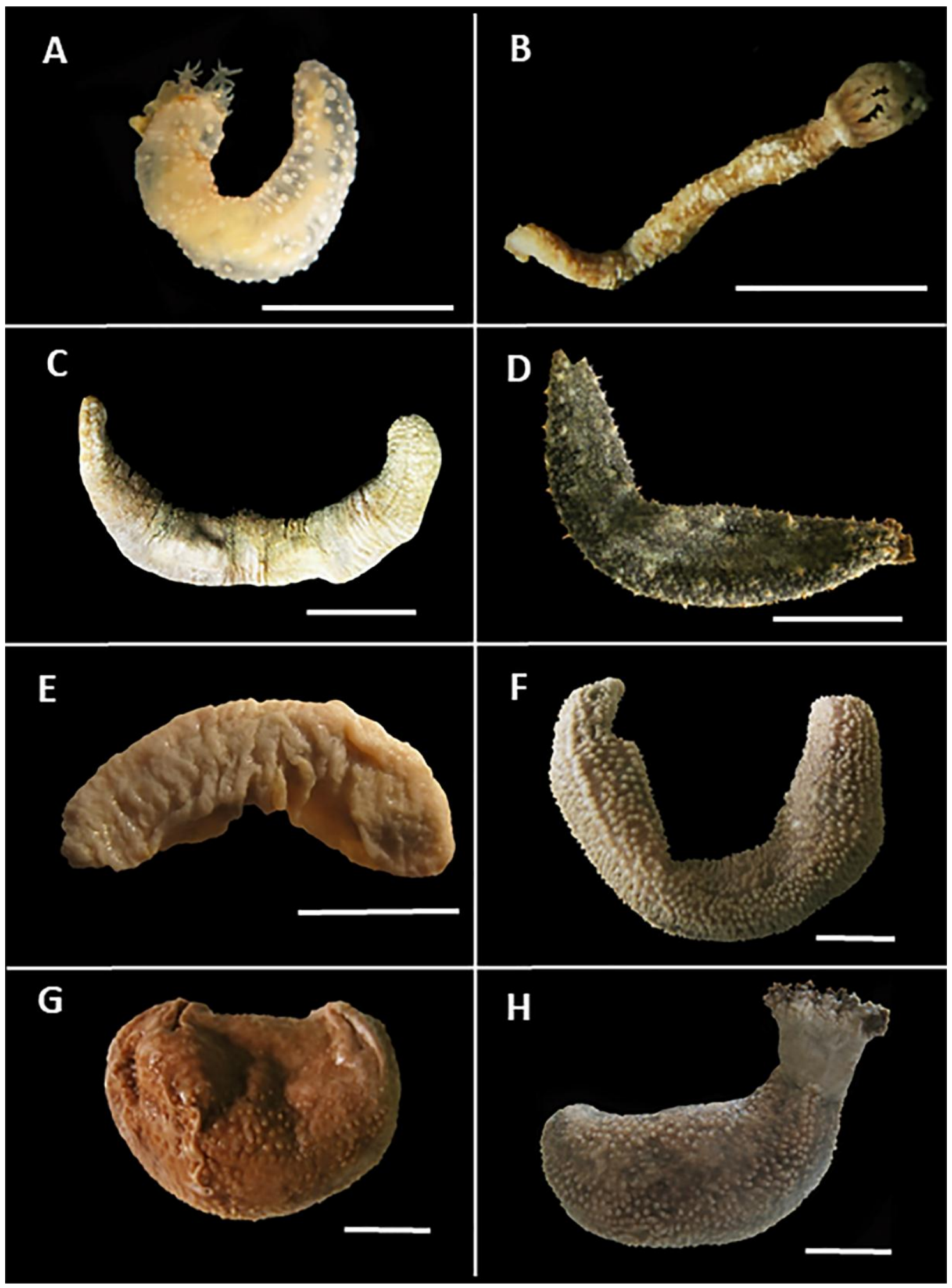

Figure 1. Holothurians on the coast of Paraíba: A. Chiridota rotífera (UFPB/ECH-2039); B. Synaptula hydriformis (UFPB/ECH-1554); C. Holothuria (Thymiosycia) arenicola (UFPB/ECH-697); D. Holothuria (Halodeima) grisea (UFPB/ECH-1222); E. Holothuria (Cystipus) pseudofossor (UFPB/ECH-2070); F. Thyonidium seguroensis (UFPB/ECH-1217); G. Parathyone suspecta (UFPB/ECH-1499); H. Parathyone braziliensis (UFPB/ECH-1063). Scale bars: A-B, E-H. 1 cm; C-D. 4 cm. 
Diagnosis: Length up to $10 \mathrm{~cm}$, with 20 dendritic tentacles. Color dark-brown to greenish-brown, with whitish tube feet. All ossicles in body wall are of the table type with four short pillars, that present about 12 marginal and four holes with rounded margins (Hendler et al. 1995).

Geographic distribution: Golfo do Mexico, Jamaica, Puerto Rico, Venezuela, Brazil (Pawson et al. 2010).

Parathyone Deichmann, 1957

Parathyone suspecta (Ludwig, 1875)

(Figure 1G)

Material examined: UFPB/ECH-1061, 1 spec., Praia do Cabo Branco, João Pessoa, PB; UFPB/ECH1499, 1 spec., Praia do Cabo Branco, João Pessoa, PB; UFPB/ECH-1216, 1 spec., Recife de Areia Vermelha, Cabedelo, PB.

Type locality: Barbados (Deichmann 1930).

Diagnosis: Body globose, with anterior end tapering, measuring up to about $7 \mathrm{~cm}$. Tegument smooth and thick. Ten tentacles of similar size, strongly branched. Tube feet present on radia and interradia. Ossicles of body wall in the shape of nodulated buttons or baskets, and tentacles with perforated bars at tips. Ossicles of introvert in the shape of buttons with nodules of variable size (Laguarda-Figueras et al. 2001).

Geographic distribution: Antilles, Colombia, and Brazil (Hendler et al. 1995).

\title{
Ocnus Forbes \& Goodsir in Forbes, 1841 \\ Ocnus braziliensis (Verrill, 1868)
}

(Figure 1H)

Material examined: UFPB/ECH-1063, 1 spec., Praia do Cabo Branco, João Pessoa, PB.

Type locality: Abrolhos Reefs, Bahia (Verrill 1868).

Diagnosis: Body fusiform, oval when contracted. Ten tentacles, the two ventral smaller. Skin thin, podia small, numerous, placed irregularly. Color whitish-grey. Calcareous ring composed of simple elements with short posterior projections. Ossicles are plates, buttons and baskets (Tommasi 1969).

Geographic distribution: Coast of Brazil, in shallow water (Tommasi 1969).

\author{
Phyllophoridae Östergren, 1907 \\ Pentamera Ayres, 1852 \\ Pentamera paraibanensis Prata \& Christoffersen, 2016
}

(Figure 2A)

Material examined: UFPB/ECH-2088, 6 spec., 06 59'01"S, 3447'23"W, PB, 07.iii.2006; UFPB/ECH141, 100 spec., 06 59'01"S, 3447'23"W, João Pessoa, PB; UFPB/ECH-148, 4 spec., 06 59'00"S, $34^{\circ} 46^{\prime} 41^{\prime \prime}$, João Pessoa, PB; UFPB/ECH-1684, 1 spec., 06059'01"S, 34045'12"W, João Pessoa, PB; UFPB/ECH-145, 86 spec., 0701'02"S, 3447'55"W, João Pessoa, PB; UFPB/ECH-149, 2 spec., $07^{\circ} 01^{\prime} 00^{\prime \prime S}, 34^{\circ} 46^{\prime} 02^{\prime \prime} \mathrm{W}$, João Pessoa, PB; UFPB/ECH-140, 1655 spec., 0703'50"S, 3447'19"W, João Pessoa, PB; UFPB/ECH-143, 400 spec., 07 03'50"S, 3447'19"W, João Pessoa, PB; UFPB/ECH-150, 31 spec., 07 03'48"S, 34 45'10"W, João Pessoa, PB; UFPB/ECH-153, 31 spec., 07 03'49"S, 3443'12"W, João Pessoa, PB; UFPB/ECH-204, 42 spec., 07 04'24.4"S; 3447'49"W, João Pessoa, PB; UFPB/ECH- 
858, 23 spec., 077'25.2"S, 346'35.0"W, João Pessoa, PB; UFPB/ECH-857, 1 spec., 07'8'28.836"S, 34'46'34.118"W, João Pessoa, PB; UFPB/ECH-2087, 1 spec., 07003'49"S, 3447'19"W, João Pessoa, PB; UFPB/ECH-205, 1 spec., Picãozinho, João Pessoa, PB; UFPB/ECH-2089, 1 spec., 07 03'50"S, $34^{\circ} 47^{\prime} 19^{\prime \prime W}, \mathrm{~PB}$; UFPB/ECH-2072, 1 spec., 07 43'09"S, 3445'00"W, PB; UFPB/ECH-2068, 1 spec., Praia de Coqueirinho, Município Conde, PB; UFPB/ECH-2058, 2 spec., 07 $07^{\prime} 00^{\prime \prime S}, 34^{\circ} 43^{\prime} 54^{\prime \prime} \mathrm{W}, \mathrm{PB}$; UFPB/ECH-2059, 1 spec., 07 $07^{\prime} 00^{\prime \prime S}, 34^{\circ} 43^{\prime} 54^{\prime \prime} \mathrm{W}, \mathrm{PB}$; UFPB/ECH-2061, 4 spec., 07 $03^{\circ} 48^{\prime \prime} \mathrm{S}$, $34^{\circ} 45^{\prime} 10^{\prime \prime W}, \mathrm{~PB}$; UFPB/ECH-2053, 5 spec., 0705'05"S, 344ㄴ'21"W, PB; UFPB/ECH-2057, 1 spec., Recifes em frente ao Iate Club da Paraíba, Praia do Bessa, PB; UFPB/ECH-2048, 1 spec., $07^{\circ} 05^{\prime} 05^{\prime \prime S}, 34^{\circ} 44^{\prime} 21^{\prime \prime W}, \mathrm{~PB}$; UFPB/ECH-2049, 50 spec., 0705'01"S, 344ㄴ'56"W, PB; UFPB/ECH-2052, 15 spec., 07 05'S, PB; UFPB/ECH-2037, 1 spec., Ponta do Cabo Branco, João Pessoa, PB; UFPB/ECH-2038, 5 spec., $07^{\circ} 03^{\prime} 48^{\prime \prime} \mathrm{S}, 34^{\circ} 45^{\prime} \mathrm{W}, \mathrm{PB}$; UFPB/ECH-2033, 13 spec., 07 $011^{\prime} 02^{\prime \prime} \mathrm{S}$, $34^{\circ} 47^{\prime} 55^{\prime \prime W}, \mathrm{~PB}$; UFPB/ECH-2030, 93 spec., 07 05'01"S, 34 47'56"W, PB; UFPB/ECH-2031, 226 spec., $07^{\circ} 05^{\prime} 59^{\prime \prime S}, 34^{\circ} 46^{\prime} 04^{\prime \prime} \mathrm{W}, \mathrm{PB}$; UFPB/ECH-1683, 14 spec., 07005'05.1"S, 3444'21"W, PB.

Type locality: João Pessoa, state of Paraiba, Brazil, 07 05'01"S, 3447'56"W, $10 \mathrm{~m}$ (Prata \& Christoffersen 2016).

Diagnosis: Body small, length up to $7 \mathrm{~mm}$, anterior and posterior ends slightly upturned. Color brown in life and in alcohol, tube feet light brown to white. Tube feet only in the radii. Ten branched tentacles, two ventral ones smaller. Skin thin, smooth. Body wall ossicles comprise oval tables with four central holes, sometimes more elongated and also with smaller holes marginally and smooth multilocular plates; spire low, with two short pillars ending in 2-3 blunt teeth. Tube feet with supporting plates, curved support tables of variable height, and endplates. Tentacles with rosettes and rods. Introvert with rosettes (Prata \& Christoffersen 2016).

Geographic distribution: Brazil (Prata \& Christoffersen 2016).

\section{Stolus Selenka, 1867 \\ Stolus cognatus (Lampert, 1885) \\ (Figure 2B)}

Material examined: UFPB/ECH-854, 1 spec., Praia do Cabo Branco, João Pessoa, PB; UFPB/ECH-

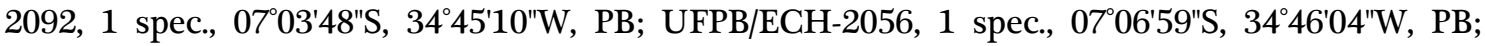
UFPB/ECH-2014, 1 spec., Recife de Areia Vermelha, Cabedelo, PB; UFPB/ECH-1944, 1 spec., Praia Formosa, Cabedelo, PB; UFPB/ECH-1945, 1 spec., Recifes em frente à Ponta de Campina, Praia do Poço, Cabedelo, PB; UFPB/ECH-1946, 1 spec., Praia do Cabo Branco, João Pessoa, PB; UFPB/ECH-1503, 1 spec., Praia do Cabo Branco, João Pessoa, PB; UFPB/ECH-155, 3 spec., Praia do Cabo Branco, João Pessoa, PB; UFPB/ECH-1066, 3 spec., Praia do Cabo Branco, João Pessoa, PB; UFPB/ECH-1073, 1 spec., Ponta do Seixas, João Pessoa, PB; UFPB/ECH-1954, 1 spec., Recife do Seixas, João Pessoa, PB; UFPB/ECH-1219, 1 spec., Barra de Camaratuba, Mataraca, PB; UFPB/ECH-1065, 1 spec., Praia do Cabo Branco, João Pessoa, PB.

Type locality: Fernando de Noronha, Pernambuco (Deichmann 1930).

Diagnosis: Body curved, with both ends tapering. The anterior end is narrower than the posterior end. Tentacles dendritic, small, similar in size, barely visible. The skin is rough due to the large quantity of ossicles. Color variable, sometimes pale with some dark spots distributed regularly or irregularly. Tube feet in double rows over entire body surface. Papillae absent (Caycedo 1978).

Geographic distribution: Florida, Antilles, Venezuela and Brazil (Hendler et al. 1995). 


\section{Thyone Jaeger, 1833}

Thyone pawsoni Tommasi, 1972

\section{(Figure 2C)}

Material examined: UFPB/ECH-1992, 1 spec., Recifes do Seixas, João Pessoa, PB; UFPB/ECH2119, 1 spec., 0703'50"S, 3447'19"W, PB.

Type locality: Gulf of Venezuela, Station “Oregon” 5679 (Tommasi 1972).

Diagnosis: Body fusiform, about $6 \mathrm{~cm}$ long, tapering into rounded tips. Color white to light brown, with dark spots. Numerous tube feet scattered over entire body. Tube feet become contracted in preserved specimens. Ten tentacles, two ventral smaller. Tables present in body wall, with four holes and spire ending in a single spine (Pawson et al. 2010).

Geographic distribution: North Carolina to Florida, eastern Gulf of Mexico, Venezuela and Brazil (Pawson et al. 2010; Martins et al. 2012a).

\section{Thyone pseudofusus Deichmann, 1930}

(Figure 2D)

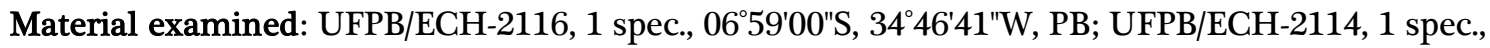
$07^{\circ} 01^{\prime} 00^{\prime \prime S}, 34^{\circ} 46^{\prime} 32^{\prime \prime W}, \mathrm{~PB}$; UFPB/ECH-2117, 1 spec., 07 03'50"S, 344ㄴ'19"W, PB; UFPB/ECH-2118, 1 spec., $07^{\circ} 03^{\prime} 50^{\prime \prime S}, 34^{\circ} 47^{\prime} 19^{\prime \prime} \mathrm{W}, \mathrm{PB}$.

Type locality: Yucatan, Station “Albatross” 2362 (Deichmann 1930).

Diagnosis: Body fusiform at ends, reaching about $2 \mathrm{~cm}$ in length, with mouth and anus directed dorsally. Color white. Tube feet in double rows along radia, irregular along interradia, particularly numerous in ventral region. Ten tentacles, the two ventral ones smaller. Body wall with thick tables with four perforations and spire short, ending in several teeth (Pawson et al. 2010).

Geographic distribution: North Carolina to northern Florida, Gulf of Mexico, Yucatan, Panama, Colombia, Antilles and Brazil (Pawson et al. 2010).

\section{Psolidae Perrier, 1902 \\ Lissothuria Verrill, 1867 \\ Lissothuria braziliensis (Théel, 1886)}

(Figure 2E)

Material examined: UFPB/ECH-1292, 1 spec., Praia do Cabo Branco, João Pessoa, PB.

Type locality: Porto Seguro, Bahia (Théel 1886).

Diagnosis: Dorsal scale completely covered. Surface deposits represent hourglass-shaped perforated plates and tables. Deposits on sole are perforated plates, which are smooth, but with elevated marginal projections. Tentacles with rosettes (Pawson 1967).

Geographic distribution: Antilles to Brazil (Tommasi 1969). 


\title{
Pseudothyone belli (Ludwig, 1887)
}

(Figure 2F)

Material examined: UFPB/ECH-2094, 2 spec., Praia do Bessa, PB; UFPB/ECH-2095, 4 spec., $06^{\circ} 59^{\prime} 01^{\prime \prime S}, 34^{\circ} 47^{\prime 23}$ "W, PB; UFPB/ECH-2096, 10 spec., 0704'24.4"S, 34 47'49"W, PB; UFPB/ECH2097, 1 spec., 07 01'02"S, 3447'35"W, PB; UFPB/ECH-2098, 1 spec., 07 03'18"S, 34 45'10"W, PB; UFPB/ECH-2099, 1 spec., 0703'50"S, 3447'19"W, PB; UFPB/ECH-2043, 1 spec., Ponta do Cabo Branco, João Pessoa, PB; UFPB/ECH-2044, 1 spec., 0704'26"S, 34049'51"W, PB; UFPB/ECH-2016, 1 spec., 0706'59"S, 3446'04"W, PB; UFPB/ECH-1990, 1 spec., Atolzinho, Praia do Bessa, João Pessoa, PB; UFPB/ECH-1959, 1 spec., Recife em frente ao Iate, praia do Bessa, PB; UFPB/ECH-146, 8 spec., 07 01'00"S, 3446'02"W, João Pessoa, PB; UFPB/ECH-144, 14 spec., 0703'50"S, 3447'19"W, João Pessoa, PB.

Type locality: Abrolhos, Bahia (Deichmann 1930).

Diagnosis: A small, burrowing species, reaching about $5 \mathrm{~cm}$ in length. Color a dirty-white, with brown dots, tips of tube feet delimited by a brown ring. In young specimens, there are many grey spots, making the animal appear black. Body cylindrical, curved. Tube feet numerous, spread over whole body. Body wall rigid, with ossicles consisting of buttons with nodules. Support tables occur in the tube feet (Pawson et al. 2010).

Geographic distribution: Bermudas, Gulf of Mexico, Antilles, Panama, Puerto Rico, Brazil (Pawson et al. 2010).

Coronatum Martins \& Souto, 2012

Coronatum baiensis Martins \& Souto, 2012

(Figure 2G)

Material examined: UFPB/ECH-2151, 13 spec., 07 06'59"S, 34 46'04"W, PB; UFPB/ECH-2153, 1 spec., 07 01'02"S, 3447'55"W, PB; UFPB/ECH-2154, 22 spec., 07 05'01"S, 34 47'56"W, PB; UFPB/ECH-2155, 4 spec., 0703'50"S, 34²7'19"W, PB; UFPB/ECH-2156, 16 spec., 07 06'59"S, $34^{\circ} 46^{\prime} 04^{\prime \prime}$, PB; UFPB/ECH-2157, 24 spec., 0705'59"S, 3446'04" W, PB.

Type locality: Amaralina Beach, Salvador, Bahia, Brazil (Martins et al. 2012b).

Diagnosis: With 10 tentacles, the two ventral ones smaller. Tube feet spread over entire body. Body wall ossicles are tables with two pillars, lobed margins, and short spire, ending in numerous teeth, podia with support towers and introvert with tables and rosettes (Martins et al. 2012b).

Geographic distribution: Brazilian coast, from the littoral of Salvador (Martins et al. 2012b) to Paraíba.

\author{
Euthyonidiella Heding \& Panning, 1954 \\ Euthyonidiella occidentalis Ludwig, 1875
}

(Figure 2H)

Material examined: UFPB/ECH-2105, 1 spec., Ponta do Seixas, João Pessoa, PB; UFPB/ECH-2106, 1 spec., 0703'50"S, 3447'19"W, PB; UFPB/ECH-2107, 1 spec., 07²8'01"S, 34 48'47"W, Praia de Pitimbú, Município Pitimbú, PB; UFPB/ECH-2108, 1 spec., 0701'02"S, 34 47'55"W, PB; UFPB/ECH2109, 1 spec., 0704'24.4"S, 3447'49"W, PB; UFPB/ECH-2110, 2 spec., Ponta do Seixas, João Pessoa, PB; UFPB/ECH-2112, 1 spec., Praia do Cabo Branco, João Pessoa, PB; UFPB/ECH-2067, 1 spec., 07 06'59"S, 3446'04"W, PB; UFPB/ECH-2063, 1 spec., 0703'51"S, 3449'51"W, PB; UFPB/ECH-2041, 
1 spec., 0707'00"S, 3443'54"W, PB; UFPB/ECH-2029, 1 spec., Praia do Cabo Branco, João Pessoa, PB; UFPB/ECH-2023, 1 spec., Ponta do Cabo Branco, João Pessoa, PB; UFPB/ECH-2018, 1 spec., 07 06'59"S, 3446'04"W, PB; UFPB/ECH-2020, 1 spec., Praia do Cabo Branco, João Pessoa, PB; UFPB

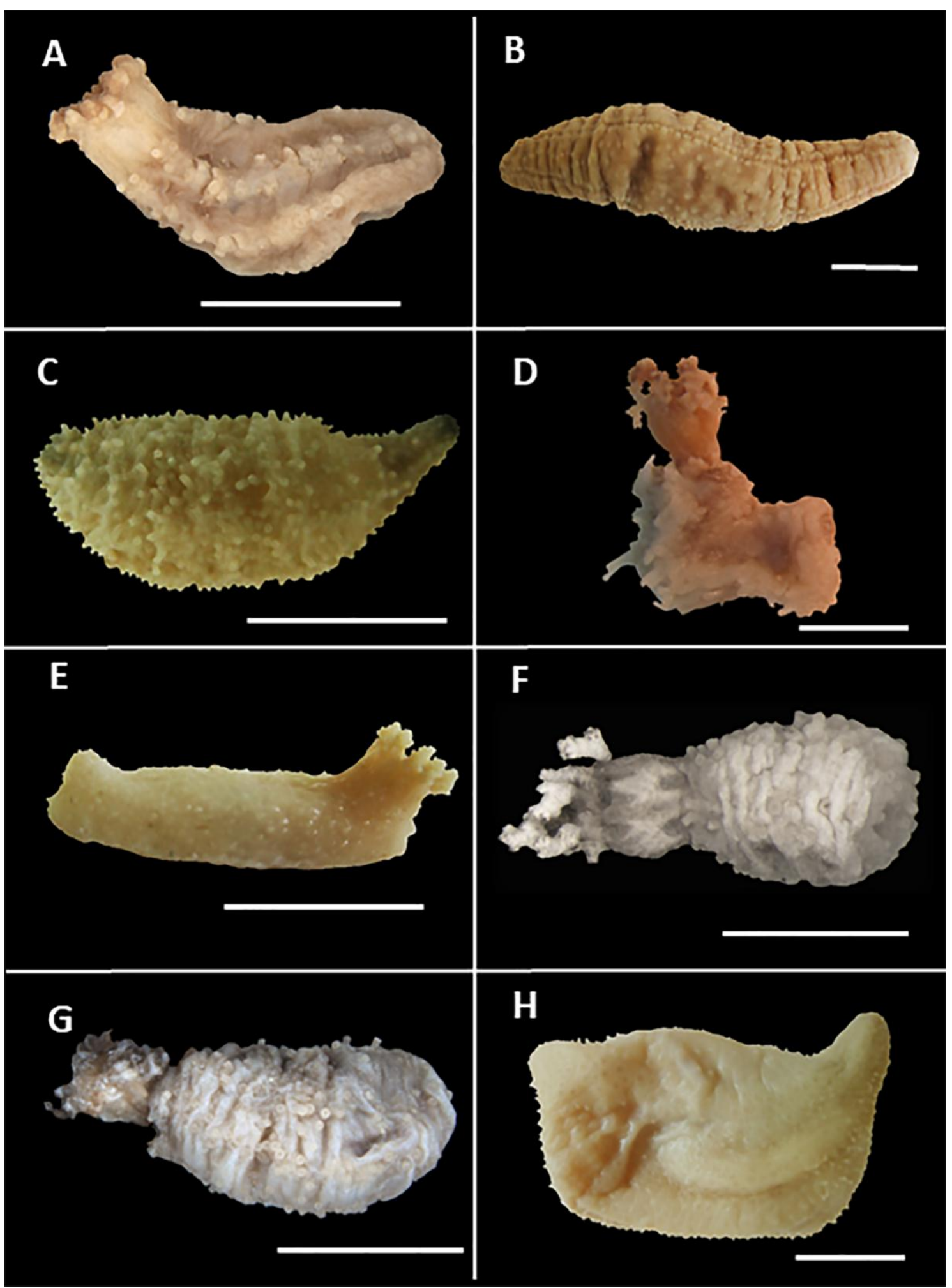

Figure 2. Holothurians on the coast of Paraíba: A. Pentamera paraibanensis (UFPB/ECH-857); B. Stolus cognatus (UFPB/ECH-1954); C. Thyone pawsoni (UFPB/ECH-1992); D. Thyone pseudofusus (UFPB/ECH2116); E. Lissothuria braziliensis (UFPB/ECH-1292); F. Pseudothyone belli (UFPB/ECH-2099); G. Coronatum baiensis (UFPB/ECH-2153); H. Euthyonidiella occidentalis (UFPB/ECH-2112). Scale bars: A, D, F-G. $0.5 \mathrm{~mm}$; B-C, E, H. $1 \mathrm{~cm}$. 
/ECH-1996, 1 spec., Praia do Cabo Branco, João Pessoa, PB; UFPB/ECH-1963, 1 spec., Recife de São Gonçalo, João Pessoa, PB; UFPB/ECH-1608, 1 spec., Recife de Areia Vermelha, Cabedelo, PB; UFPB/ECH-1504, 1 spec., Praia do Maceiozinho, Jacumã, Conde, PB; UFPB/ECH-1221, 2 spec., Recife de Areia Vermelha, Cabedelo, PB; UFPB/ECH-1218, 1 spec., Barra de Camaratuba, Mataraca, PB; UFPB/ECH-1064, 1 spec., Praia do Cabo Branco, João Pessoa, PB; UFPB/ECH-866, 1 spec., Praia do Cabo Branco, João Pessoa, PB; UFPB/ECH-855, 1 spec., Praia do Cabo Branco, João Pessoa, PB; UFPB/ECH-1995, 1 spec., Recifes em frente à Ponta de Campina, Praia do Poço, Cabedelo, PB; UFPB/ECH-434, 1 spec., Praia do Cabo Branco, João Pessoa, PB; UFPB/ECH-435, 1 spec., Praia do Cabo Branco, João Pessoa, PB; UFPB/ECH-1067, 1 spec., Praia do Cabo Branco, João Pessoa, PB; UFPB/ECH-152, 2 spec., João Pessoa, PB.

Type locality: Surinam (Deichmann 1930).

Diagnosis: May attain about $10 \mathrm{~cm}$, barrel-shape. Body cylindrical, "U"-shaped, slightly tapering towards extremities. Twenty alternating large and small tentacles, podia arranged along the ambulacral zone and spread uniformly over entire body. Ossicles consisting of uniform tables. In life color yellow, orange or dark brown (Miller \& Pawson 1984).

Geographic distribution: Florida, Puerto Rico, Antilles, Aruba, Trinidad, Surinam and Brazil (Hendler et al. 1995).

\section{Discussion}

A review of the literature permits an extended analysis of the biodiversity and distribution of the holothurians studied on the coast of Paraíba. Thirteen species studied here are spread over a broad geographic area from the western Atlantic. Thyone pawsoni, T. pseudofusus and Pseudothyone belli have distributions shared between the North Carolina/Bermudas to Brazil, extending the southern limit of distribution to Brazil (Pawson et al. 2010; Martins et al. 2012a). Most of the species identified here (e.g., Chiridota rotifera, Synaptula hydriformis, Holothuria (T.) arenicola, Thyonidium seguroensis, Parathyone suspecta, Stolus cognatus, Euthyonidiella occidentalis) are distributed from the Florida/West Indies to Brazil (Tommasi 1969, 1972; Hendler et al. 1995; Pawson et al. 2010).

Despite the wide distribution of these holothurians in the western Atlantic, there are wide gaps between the reported localities, especially between the West Indies and the Atlantic coast of South America. Among the species analyzed herein, only Holothuria (Halodeima) grisea Selenka, 1867 has been recognized as Amphi-Atlantic and found also in the eastern Atlantic off West Africa (Pawson et al. 2010). To the best of our knowledge, there has been no previous information published on the occurrence of Coronatum baiensis, Pentamera paraibanensis and Ocnus braziliensis along the Brazilian coast (Tommasi 1969; Martins et al. 2012a,b; Prata \& Christoffersen 2016). Pentamera paraibanensis is only known from its type locality on the coast of Paraíba (Prata \& Christoffersen 2016).

The species Chiridota rotifera and Holothuria (Halodeima) grisea were the most usual and most widely distributed, occurring along the entire coastline. According the previous studies (see Brito 1962; Lima-Verde 1969; Gondim et al. 2008, 2011; Prata et al. 2014a, 2016b), only 11 species were recorded for the coast of the State of Paraíba. The species Thyonidium seguroensis, Ocnus braziliensis, Thyone pawsoni, T. pseudofusus and Coronatum baiensis are now added to this occurrence list.

Pentamera paraibanensis was mistakenly identified as Phyllophorus comunis (Forbes, 1841) and Cucumaria pulcherrima (Ayres, 1854) (currently Pentamera pulcherrima Ayres, 1852) by Gondim et al. (2008: 154, 2011:7) for the coast of Paraíba. These specimens are deposited in the CIPY and were reanalyzed.

This study represents the first checklist of Holothuroidea for the coast of Paraíba. The knowledge of a little studied group is increased based on the present study from the 
southwestern Atlantic. Holothurians are among the echinoderms still very poorly studied in northeastern Brazil. Most known holothurians of this region occur on the continental shelf (Tommasi 1969; Martins et al. 2012a,b; Prata \& Christoffersen 2016) and no one study have been recorded for deep waters.

Currently, the holothurian fauna of the coast of Paraíba can be recognized as rather underestimated. Certainly, there is considerable species richness yet to be studied, especially described and recorded for the coast of Brazil, particularly in the coastal zone of the northeastern region. Adequate knowledge on holothurians inhabiting the coast of Paraíba will serve as a basis for future studies, including analysis of environmental monitoring and faunal conservation.

\section{Acknowledgments}

J. Prata acknowledges the Master in Sciences Scholarship received from Brazilian Federal Agency for Support and Evaluation of Graduate Education (CAPES). M. L. Christoffersen was supported by a productivity scholarship from National Council for Scientific and Technological Development (CNPq). The anonymous reviewers are thanked for critically reviewing the manuscript.

\section{References}

Amaral A.C.Z. \& Jablonsk S. (2005) Conservação da biodiversidade marinha e costeira no Brasil. Megadiversidade, 1(1): 43-51.

Ancona-Lopez A.A. \& Sawaya, P. (1955) Holotúrias de Recife. Ciência e Cultura, 7: 166.

Appeltans W., Bouchet P., Boxshall G.A., Broyer C., Voogd N.J., Gordon D.P., Hoeksema B.W., Horton T., Kennedy M., Mees J., Poore G.C.B., Read G., Stöhr S., Walter T.C. \& Costello M.J. (2012) World register of marine species. Available from: http://www.marinespecies.org (Accessed on 14.02.2017).

Araújo P.G., Miranda G.E.C. \& Kanagawa A.I. (2008) Repartição espacial da comunidade macrobêntica dos recifes da APA da Barra do Rio Mamanguape, Paraíba, Brasil. Revista Nordestina de Biologia, 19(1): 29-50.

Brito I.M. (1962) Ensaio de catálogo dos equinodermas do Brasil. Avulso n 13, Centro de Estudos Zoológicos, 11: 1-11.

Caycedo I.E. (1978) Holothuroidea (Echinodermata) de águas someras em la costa norte de Colombia. Anais do Instituto de Investigações marinhas, 10: 149-198.

Costa C., Sassi R. \& Costa M. (2007) Recifes Costeiros da Paraíba, Brasil: usos, impactos e necessidades de manejo no contexto da sustentabilidade. Gaia Scientia, 1(1): 37-45.

De Assis J.E., Samiguel C.A., Brito R.J., Christoffersen M.L. \& Santos A.S. (2012) Polychaetous annelids from the coast of Paraíba state, Brazil. Revista Nordestina de Biologia, 21: 3-44.

Deichmann E. (1930) The Holothurians of the western part of the Atlantic Ocean. Bulletin of the Museum of Comparative Zoology at Harvard College, 71(3): 42-276.

Deichmann E. (1958) The Holothuroidea collected by the Velero III and IV during the years 1932 to 1954. Part II. Apsidochirota. Allan Hancock Pacific Expeditions, 11(2): 239-349.

Deichmann E. (1963) Shallow water holothurians known from the Caribbean waters. Studies Fauna Curaçao, Caribbean Islands, 63: 100-118.

Gondim A.I., Lacouth P., Alonso C. \& Manso C.L.C. (2008) Echinodermata da Praia do Cabo Branco, João Pessoa, Paraíba, Brasil. Biota Neotropica, 8(2): 151-159.

Gondim A.I., Dias T.L.P., Campos F.F., Alonso C. \& Christoffersen M.L. (2011) Macrofauna bêntica do Parque Estadual Marinho de Areia Vermelha, Cabedelo, Paraíba, Brasil. Biota Neotropica, 11(2): 75-85.

Heezen B.C. \& Hollister C.D. (1971) The face of the deep. New York, London, Toronto: Oxford University Press. 659 p. 
Hendler G., Miller J.E., Pawson D.L. \& Kier P.M. (1995) Sea Stars, Sea Urchins and allies: Echinoderms of Florida and the Caribbean. Washington: Smithsonian Institution Press. 392 p.

Laguarda-Figueras A., Solís-Marín F.A., Durán-Gonzalez A., Pliego P.H. \& García R. del V. (2001) Holothuroideos (Echinodermata: Holothuroidea) del Caribe Mexicano: Puerto Morelos, Quintana Roo, México. Avicennia, 14: 7-46.

Lessius H.A. (2005) Echinoids of the Pacific Waters of Panama: Status of knowledge and new records echinoids of the Pacific waters of Panama. Revista de Biología Tropical, 53: 147-170.

Lesueur C.A. (1824). Description of several new species of Holothuria. Journal Academy Natural Sciences, 4: 155-163.

Lima-Verde J.S. (1969) Primeira contribuição ao inventário dos Echinodermas do nordeste Brasileiro. Arquivos de Ciências do Mar, 9(1): 9-13.

Lima S.F.B., Queiroz V., Bravo de Laguna I.H. \& Mioso R. (2014) New host for Dissodactylus crinitichelis (Decapoda, Pinnotheridae): First record of occurrence on Mellita quinquiesperforata (Echinodermata, Echinoidea) (Decapoda; Echinodermata). Spixiana, 37(1): 61-68.

Lima S.F.B., Lucena R.A., Santos G.M., Souza J.W., Christoffersen M.L., Guimarães C.R. \& Oliveira G.S. (2017) Inventory of mollusks from the estuary of the Paraíba River in northeastern Brazil. Biota Neotropica, 17(1): 1-12 (e20160239). doi: 10.1590/1676-0611-BN-2016-0239

Ludwig H. (1881) Über eine lebendiggebarende Synaptide und zwei andere holothurienarten des Brasilianischen kuste. Archives Italiennes de Biologie, 2: 41-58. 1881.

Martins L. \& Souto C. (2015) Taxonomic review of four western Atlantic dendrochirotids (Holothuroidea) with the description of a new Brazilian cucumariid species and designation of neotypes. Zootaxa, 3919: 362-374.

Martins L., Souto C., Braga J. \& Tavares M. (2017) Echinoidea and Holothuroidea (Echinodermata) of the Trindade and Martin Vaz Archipelago, off Brazil, with new records and remarks on taxonomy and species composition. Journal of the Marine Biological Association of the United Kingdom: 1-35. doi: 10.1017/S0025315416001569

Martins L., Souto C. \& Menegola C. (2012a) First record of Holothuria (Theelothuria) princeps and Thyone pawsoni (Echinodermata: Holothuroidea) in the South Atlantic Ocean. Marine Biodiversity Records, 5: 1-6. doi: 10.1017/S1755267212000796

Martins L., Souto C. \& Menegola C. (2012b) A new genus and new species of Sclerodactylidae (Holothuroidea: Dendrochirotida) from the south-western Atlantic coast. Zootaxa, 3506: 5462.

Miller J.E. \& Pawson D.L. (1984) Holothurians (Echinodermata: Holothuroidea). Memoirs of the Hourglass Cruises, 7(1): 1-79.

Moura R.B., Campos L.S. \& Esteves A.M. (2015). Hooked from the deep: a rare new species of Taeniogyrus (Holothuroidea, Chiridotidae) from the continental slope of Brazil, southwestern Atlantic. Zootaxa, 3972(4): 535-548.

Paraíba (Estado). (2000) Palácio do Governo do Estado da Paraíba. Decreto do governo estadual n. 21.263 de 28 de agosto de 2000 cria o Parque Estadual Marinho de "Areia Vermelha” e dá outras providências. João Pessoa: Diário Oficial do Estado da Paraíba, 29 ago. 2000.

Pawson D.L. (1967) The Psolid Holothurian Genus Lissothuria. Proceedings of the United States National Museum, 122(3592): 1-17.

Pawson D.L., Pawson D.J. \& King R.A. (2010) A taxonomic guide to the Echinodermata of the South Atlantic Bight, USA: 1. Sea cucumbers (Echinodermata: Holothuroidea). Zootaxa, 2449: $1-48$.

Prata J. \& Christoffersen M.L. (2012) Holothuria (Semperothuria) surinamensis Ludwig, 1875 (Echinodermata: Holothuroidea): Record of the species for the northeast coast of Brazil. CheckList, 8(4): 768-770.

Prata J. \& Christoffersen M.L. (2016) A new species of Pentamera Ayres, 1852 from the Brazilian coast (Holothuroidea, Dendrochirotida, Phyllophoridae). ZooKeys, 634: 1-14. 
Prata J., Dias T.L.P.. \& Christoffersen M.L. (2014a) Occurrence of Holothuria (Holothuria) dakarensis (Holothuroidea: Echinodermata) in the south-western Atlantic, with notes on distribution and ecology. Marine Biodiversity Records, 7: 1-6. doi: 10.1017/S175526721400004 9

Prata J., Manso C.L.C. \& Christoffersen M.L. (2014b) Aspidochirotida (Echinodermata: Holothuroidea) from the northeast coast of Brazil. Zootaxa, 3889(1): 127-150.

Rowe F.W.E. \& Doty J.E. (1977) The shallow water holothurians of Guam. Micronesica, 13(2): 217-250.

Sluiter C.P. (1910) Westindische holothurien. Zoologische Jahrbucher (Supplement), 11(2): 331342.

Théel H. (1886) Report on Holothurioidea. Reports on the results of dredging, under the Supervision of Alexander Agassiz, in the Gulf of Mexico (1877-78), in the Caribbean Sea (1879-80), and along the Eastern Coast of the United States during the Summer of 1880, by the U.S. Coast Survey Steamer "Blake". Bulletin of the Museum of Comparative Zoology, 13: $1-21$.

Tommasi L.R. (1969) Lista dos Holothuroidea recentes do Brasil. Contribuições Avulsas do Instituto Oceanográfico, 15: 1-29.

Tommasi L.R. (1972) Equinodermes da região entre o Amapá (Brasil) e a Flórida (E.U.A.). II. Echinozoa. Boletim Instituto Oceanográfico da Universidade de São Paulo, 21: 15-67.

Ventura C.R.R., Borges M., Campos L.S., Costa-Lotufo V., Freire C. A., Hadel V.F., Manso C.L.C., Silva J.R.M.C., Tavares Y. \& Tiago C.G. (2012) Echinoderm from Brazil: historical, research and the current state of biodiversity knowledge (p. 301-344). In: Alvarado J.J. \& Solís-Marín F. (Eds) Echinoderm research and diversity in Latin America. Berlin: Springer. 675 p.

Verrill A.E. (1868) Notice on the corals and echinoderms collected by Prof. C.F. Hartt at the Abrolhos reefs, Province of Bahia, Brasil, 1867. Transactions of the Connecticut Academy of Arts and Sciences, 1: 351-371. 